\title{
Three-dimensional edge transport simulations for DIII-D plasmas with resonant magnetic perturbations
}

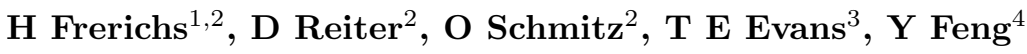 \\ ${ }^{1}$ German Research School for Simulation Sciences, Jülich, Germany \\ ${ }^{2}$ Institute for Energy Research - Plasma Physics, Forschungszentrum Jülich, \\ Germany \\ ${ }^{3}$ General Atomics, San Diego, California, CA, USA \\ ${ }^{4}$ Max-Planck Institute for Plasma Physics, Greifswald, Germany \\ E-mail: h.frerichs@fz-juelich.de
}

\begin{abstract}
In the plasma scenarios currently envisaged for ITER, edge localized modes (ELMs) are a critical issue for the wall lifetime of ITER. However, a promising technique as been found to mitigate ELMs: the application of resonant magnetic perturbations (RMPs) at the plasma edge. These introduce a complex magnetic field structure in the plasma edge, affecting also plasma transport. So far, numerical models for edge plasma transport in ELM control scenarios at DIII-D include only averaged effects of RMPs or include only heat transport. Our approach includes a self-consistent fluid treatment of particle, parallel momentum and energy transport as well as kinetic neutral production and transport. This model is implemented in the EMC3-EIRENE code which is a powerful tool to investigate $3 \mathrm{D}$ effects of RMPs on edge transport. In the present paper we apply the EMC3-EIRENE code to conditions at the DIII-D tokamak in the presence of RMPs. We demonstrate that the magnetic field structure is reflected in the plasma structure as well, in particular we investigate the pattern of particle and heat loads on the divertor target and a 3D modulation of plasma parameter in the X-point region and near the midplane on the high field side (HFS). These findings are consistent with earlier heat transport modeling results at DIII-D as well as observations and modeling results at TEXTOR.
\end{abstract}

PACS numbers: 52.65.-y, 52.65.Kj

Submitted to: Nucl. Fusion

\section{Introduction}

Presently, ITER relevant plasma scenarios are investigated at the DIII-D tokamak and also at other fusion devices. In these high-confinement (H-mode) plasmas an instability - so called edge localized mode (ELM) [1] - occurs naturally at the plasma edge, leading to high transient heat loads to the first wall and the divertor targets. Extrapolations to ITER relevant power levels indicate that this will lead to a significant reduction of ITER wall lifetime [2]. A promising technique to control ELMs is the application of resonant magnetic perturbations (RMPs) at the plasma edge, as 
successfully demonstrated at DIII-D [3, 4] and JET [5]. Recently, RMPs are under consideration for ELM mitigation in ITER.

Following earlier experiments at Tore-Supra [6, 7], RMP coils have also been installed in various other tokamaks such as TEXTOR and DIII-D, in order to actively control particle and heat transport. Modeling of the perturbed magnetic field structure in the so called vacuum approach suggests the formation of an open chaotic system at the plasma edge with remanent magnetic island chains, partially ergodic domains and short magnetic flux tubes. (figure 1). However, the detailed resulting impact of such a complex magnetic field structure on plasma transport is still ongoing research. In particular the impact on the pressure gradient in the edge transport barrier is of great interest, because this is correlated with the stabilization of peeling-balloning MHD modes considered as the cause of ELMs [8]. In present edge transport models for RMP ELM control scenarios $[9,10]$ only the magnetic flux surface averaged effects of RMPs are considered. There is, however, strong experimental evidence, e.g. at the TEXTOR $[11,12]$ and DIII-D tokamaks [13], that the structure of the 3D magnetic field topology is reflected on the plasma structure as well. The EMC3-EIRENE code $[14,15,16,17]$ is a powerful tool to investigate such $3 \mathrm{D}$ effects on edge transport, not only in stellarators but also in limiter tokamaks $[18,19,20]$, the ITER-startup-limiter configuration [21] and recently in poloidally diverted tokamaks [22]. A self-consistent fluid treatment of particle, parallel momentum and electron and ion energy transport is included as well as kinetic neutral production and transport.

In order to develop a reliable 3D edge modeling tool to enable predictions for future fusion devices, the code needs to be validatd with observations at present, ITER relevant experiments such as DIII-D. For this the EMC3-EIRENE code has been advanced to magnetic field configurations with arbitrary geometry and in particular to poloidal divertor geometry [22]. In the present paper we apply the EMC3-EIRENE code to conditions at the DIII-D tokamak, simulating the 3D effects of an RMP field with toroidal mode number $n=3$ on an ITER similar shape plasma. We demonstrate that the magnetic field structure is reflected in the plasma structure as well, leading to a significant 3D modulation of parma parameter.

The structure of this paper is as follows: First, in section 2 we give an overview on the magnetic field structure used for the transport studies later in this paper. Then in section 3 we briefly introduce the $3 \mathrm{D}$ edge transport model used for our analysis. In section 4 we analyze 3D volume effects of RMPs on plasma transport. In particular we investigate the impact of helical magnetic lobes and short magnetic flux tubes. In addition we investigate the imprints of particle and heat fluxes on the divertor target in the presence of RMPs in section 5. The influence of anomalous cross-field transport is investigated and the modeled imprint pattern compared to experimental observations. The main results are summarized in the conclusions 6 .

\section{Magnetic Field Structure}

In the following we consider an ITER similar shape (ISS) plasma at DIII-D with elongation $\kappa=1.8$ and triangularity $\bar{\delta}=\frac{1}{2}\left(\delta_{u}+\delta_{d}\right)=0.53$. The RMP field is generated by the I-coils powered by $I_{c}=4 \mathrm{kA}$ while error fields due to coil misalignements and error field correction by the C-coils are neglected. The resulting magnetic field structure is analyzed in the so called vaccuum approach, i.e. by taking an axisymmetric MHD equilibrium from the EFIT code [23] and superimposing the 3D vacuum field of the perturbation coils. 


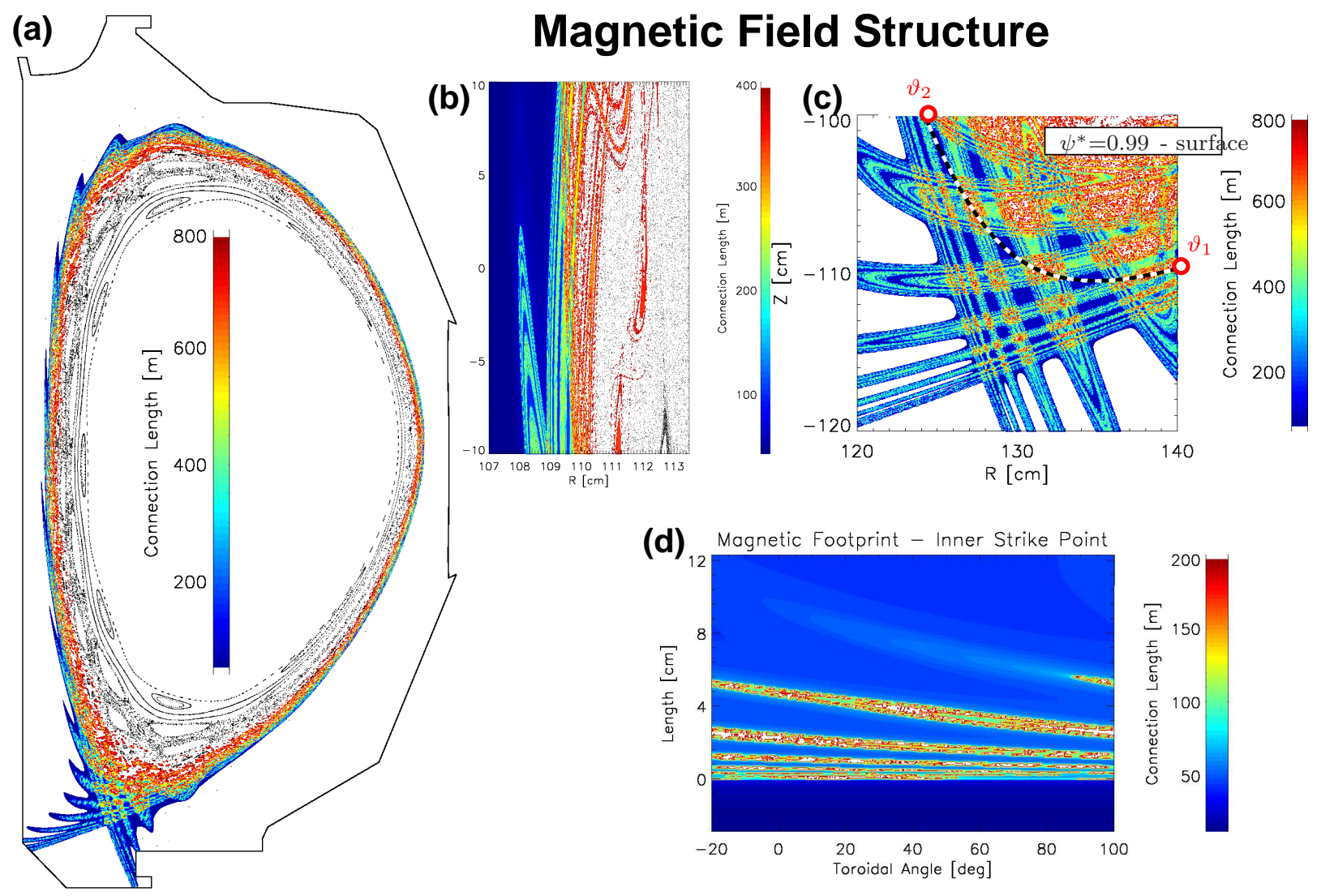

Figure 1. Magnetic field structure of a typical DIII-D plasma with ITER similar shape in the presence of an $n=3$ RMP field. The wall-to-wall connection length $L_{c}$ of magnetic field lines is indicated by color in addtion to a Poincaré plot, i.e. piercing points of field lines with a reference plane. (a) full cross-section at $\varphi=0 \mathrm{deg}$, (b) zoom of the HFS midplane region, (c) zoom of the X-point region. The position of the $\psi^{*}=0.99$-surface is marked by a white and black dashed line with the end points corresponding to poloidal angles of $\vartheta_{1}=197.4 \mathrm{deg}$ and $\vartheta_{2}=206.5 \mathrm{deg}$. (d) Magnetic footprint on the divertor target at the inner strike point.

The magnetic field structure is visualized in figure 1 by the wall-to-wall connection length $L_{c}$ of field lines and by so called Poincaré-plots, i.e. piercing points of field lines with a reference plane. The complex field structure includes magnetic island chains, partially ergodic domains (yellow, red and white regions with scattered black dots in figure 1.a-c), short magnetic fluxtubes $\left(L_{c} \lesssim 200 \mathrm{~m}\right.$, blue region in figure 1.a-c) and helical magnetic lobes guiding field lines from inside the separatrix to the divertor targets, thereby creating a helical striation pattern on targets (figure 1.d) [13, 24, 25]. These helical lobes extend into the regular Scrape-Off Layer (SOL) and are formed by the separatrix manifolds [26], also known as homoclinic tangles in perturbed nonlinear dynamical systems $[27$, p. 222]. In the following we investigate the effects of such a magnetic field structure on plasma transport. 
Three-dimensional edge transport simulations for DIII-D plasmas with resonant magnetic perturbations 4

\section{3D transport model for the edge plasma}

In the present edge plasma model we use a prescribed magnetic field structure as input for the 3D edge transport code EMC3-EIRENE. The code applies a reversible field line mapping technique [28] for a fast and accurate reconstruction of magnetic field lines from a field aligned grid. This technique has been advanced from regular structured to block-structured grids in order to allow simulation domains with nonsimple shape, such as the edge plasma in poloidally diverted tokamaks [22]. Field lines are used to define a field aligned coordinate system in which the fast parallel transport is decoupled from the small cross-field transport.

The EMC3-EIRENE code solves a set of steady state fluid equations in a 3D space of arbitrary magnetic field geometry. Balance equations for density $n$, parallel momentum $u_{\|}$and energy for electrons and ions $T_{e}$ and $T_{i}$, respectively, read:

$$
\begin{gathered}
\nabla \cdot\left[n u_{\|} \mathbf{e}_{\|}-D \underline{\mathbf{I}}_{\perp} \cdot \nabla n\right]=S_{p} \\
\nabla \cdot \mathbf{e}_{\|}\left[m_{i} n u_{\|}^{2}-\eta \mathbf{e}_{\|} \cdot \nabla u_{\|}\right] \\
-\nabla \cdot \underline{\mathbf{I}}_{\perp} \cdot D \nabla\left(m_{i} n u_{\|}\right)=-\mathbf{e}_{\|} \cdot \nabla p+S_{m} \\
\nabla \cdot \mathbf{e}_{\|}\left[\frac{5}{2} T_{e} n u_{\|}-\kappa_{e} \mathbf{e}_{\|} \cdot \nabla T_{e}\right] \\
-\nabla \cdot \underline{\mathbf{I}}_{\perp} \cdot\left[\chi_{e} n \nabla T_{e}+\frac{5}{2} T_{e} D \nabla n\right]=-k\left(T_{e}-T_{i}\right)+S_{e e} \\
\nabla \cdot \mathbf{e}_{\|}\left[\frac{5}{2} T_{i} n u_{\|}-\kappa_{i} \mathbf{e}_{\|} \cdot \nabla T_{i}\right] \\
-\nabla \cdot \underline{\mathbf{I}}_{\perp} \cdot\left[\chi_{i} n \nabla T_{i}+\frac{5}{2} T_{i} D \nabla n\right]=+k\left(T_{e}-T_{i}\right)+S_{e i}
\end{gathered}
$$

where $\mathbf{e}_{\|}$is the unit vector in diretion of the magnetic field, $\underline{\mathbf{I}}_{\perp}=\underline{\mathbf{I}}-\mathbf{e}_{\|} \mathbf{e}_{\|}$with the unit tensor $\underline{\mathbf{I}}$ and $p=n\left(T_{e}+T_{i}\right)$. For the parallel transport coefficients $\eta, \kappa_{e}, \kappa_{i}$ the classical Braginskii [29] model is assumed, while anomalous cross-field transport is taken into account by free model parameters $D, \chi_{e}, \chi_{i}$.

The EMC3 code $[14,15,16]$ applies a Monte Carlo method [16] to solve these equations for the edge plasma. Interactions with neutral particles are taken into account by sources (or sinks) in the balance equations $S_{p}, S_{m}, S_{e e}$ and $S_{e i}$, which are calculated by the kinetic neutral transport code EIRENE [17]. An iterative procedure is applied to obtain a self-consistend solution for all plasma parameters.

\section{3D effects on plasma transport}

In this section we analyze the 3D effects of RMPs on plasma transport as introduced by the magnetic field structure from figure 1 . Boundary conditions at the inner simulation boundary $\left(\psi^{*} \approx 0.77\right)$ were set to $n_{\text {in }}=1.2 \cdot 10^{19} \mathrm{~m}^{-3}$ and $P_{\text {in }}=0.9 \mathrm{MW}$ which corresponds to actual experimental values observed during the L-mode phase in DIIID discharge 132731. The complex magnetic field structure already suggests that a 
Three-dimensional edge transport simulations for DIII-D plasmas with resonant magnetic perturbations 5
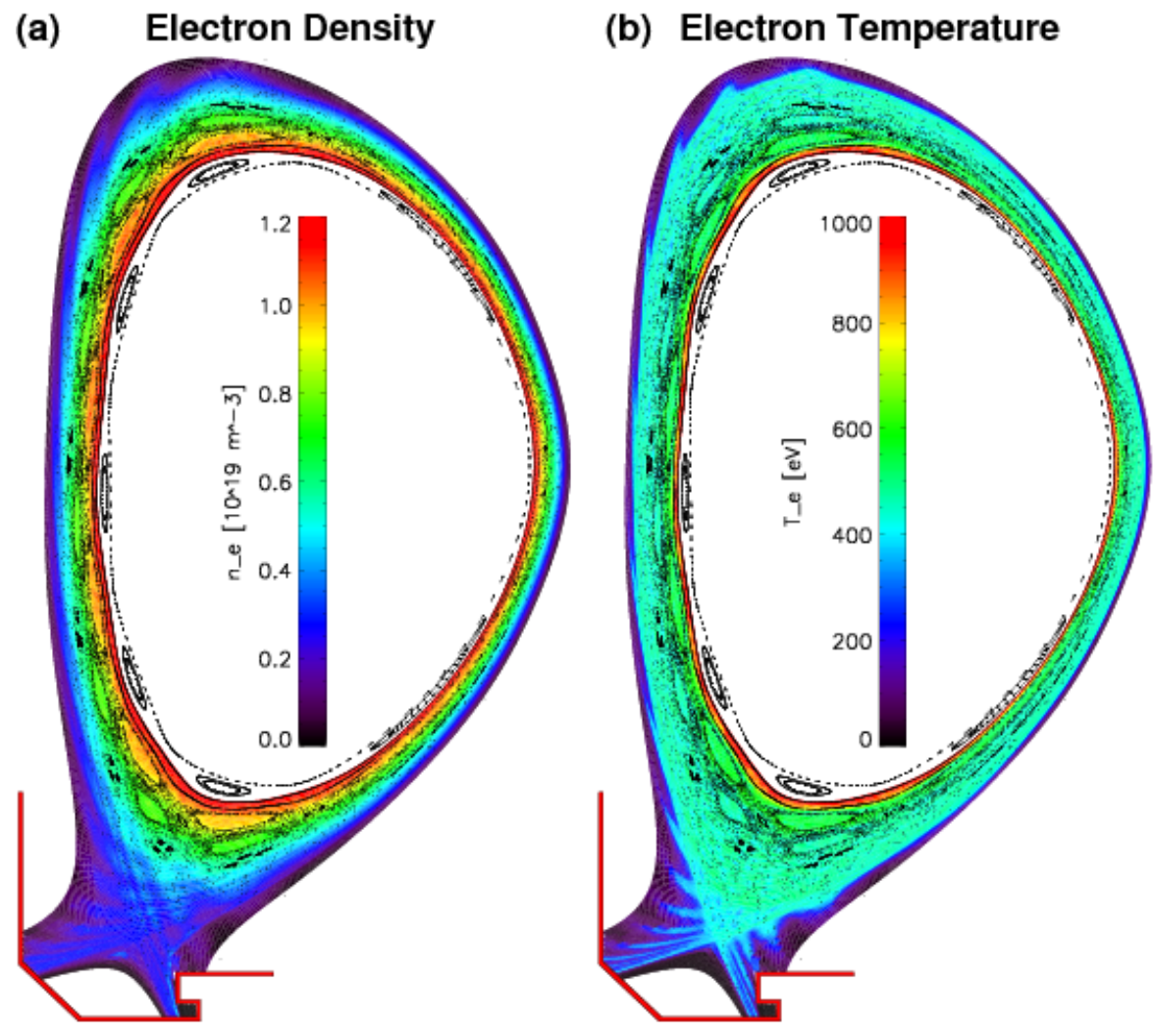

Figure 2. $2 \mathrm{D}$ cuts of the electron density $n_{e}$ (a) and electron temperature $T_{e}$ (b) at a reference plane $\varphi=0 \mathrm{deg}$. Poincaré-plots (black dots) are overlayed.

$3 \mathrm{D}$ modulation is also present in the plasma structure. The strong modulation of the complete edge plasma is demonstrated in figure 2 for the electron density $n_{e}$ and electron temperature $T_{e}$ by $2 \mathrm{D}$ cuts at a reference plane $\varphi=0 \mathrm{deg}$. Clearly, the structure of the magnetic topology (figure 1.a) is reflected in the plasma. Here we want to investigate the 3D modulation in more detail, in particular the effects of short magnetic flux tubes in the X-point region (section 4.2) and helical magnetic lobes at the HFS midplane (section 4.1).

\subsection{Helical magnetic lobes}

In the presence of RMPs an intermediate layer between confined plasma and the regular SOL exists. In this layer field lines have a finite but not too short connection length of the order $L_{c}=200-300 \mathrm{~m}$ (green - orange region in figure 1.b). This layer is bounded by the separatrix manifolds in the form of helical magnetic lobes which extend into the SOL. The helical nature of this boundary is most pronounced in the $\mathrm{X}$ point region (figure 1.c), but also clearly visible on the high field side (HFS) midplane (figure 1.b). With the EMC3-EIRENE code it is possible to investigate the detailed 

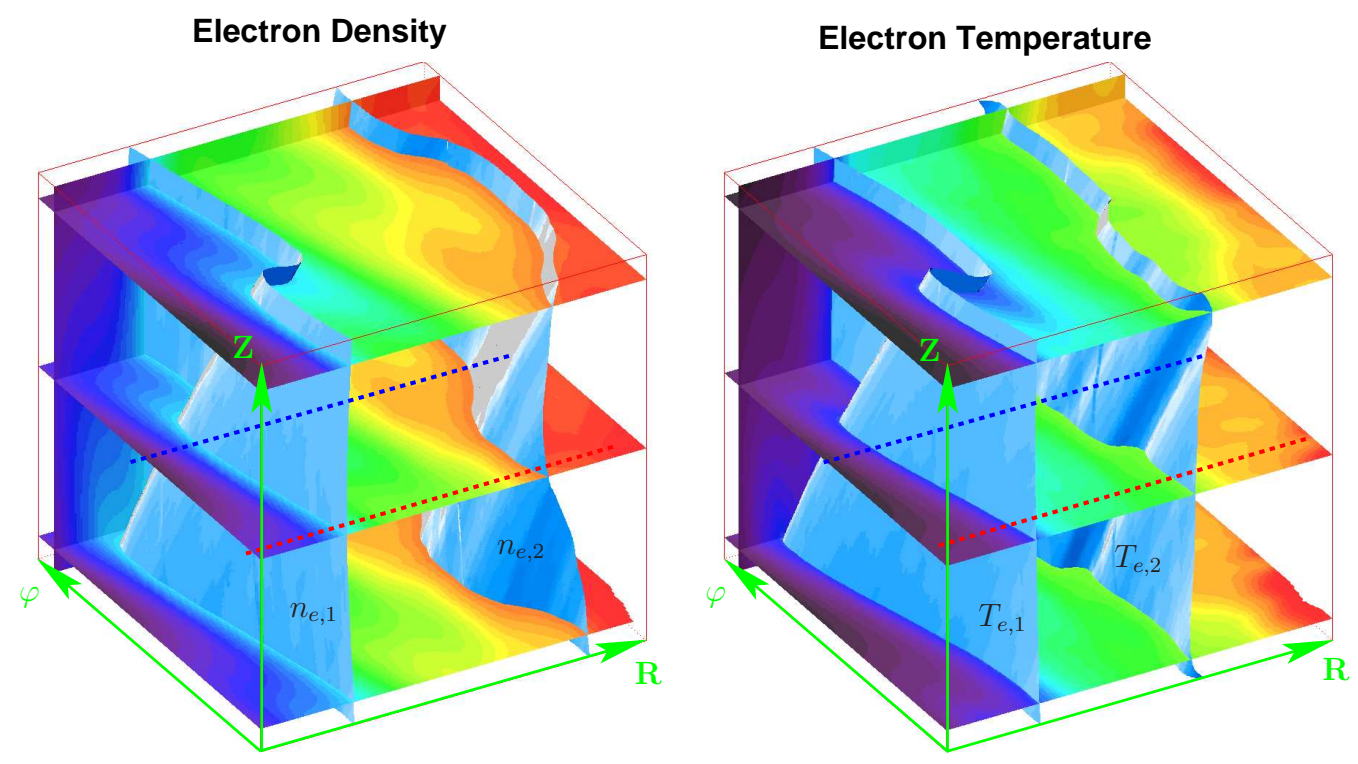

Figure 3. 3D visualization of electron density (left) and temperature (right) at the HFS midplane: $R=107 \ldots 113 \mathrm{~cm}, Z=-12 \ldots 12 \mathrm{~cm}, \varphi=$ $-20 \ldots 100 \mathrm{deg}$. Iso- $n_{e}$ surfaces are blue-shaded for $n_{e, 1}=4.5 \cdot 10^{18} \mathrm{~m}^{-3}$ and $n_{e, 2}=8.5 \cdot 10^{18} \mathrm{~m}^{-3} ;$ iso- $T_{e}$ surfaces are at $T_{e, 1}=60 \mathrm{eV}$ and $T_{e, 2}=120 \mathrm{eV}$. Red and blue dashed lines mark the positions for the profiles shown in figure 5

effects of this structure on the plasma. It is demonstrated in figure 3 and 4 that the structure of these magnetic lobes emerges also in the modeled $n_{e}$ and $T_{e}$. These figures show $3 \mathrm{D}$ visualizations of $n_{e}$ and $T_{e}$ by means of selected $2 \mathrm{D}$ cuts (rainbow coloured) and iso-parametric surfaces (blue shaded). In particular the $n_{e, 1}=4.5 \cdot 10^{19} \mathrm{~m}^{-3}$ and $T_{e, 1}=60 \mathrm{eV}$ surfaces reflect very well the structure of the helical magnetic lobes.

Hence, the midplane profiles of plasma parameter depend strongly on the toroidal position. Profiles of $n_{e}$ and $T_{e}$ are extracted in figure 5 at $\varphi_{1}=-10 \mathrm{deg}$ (red solid line) and $\varphi_{2}=50 \mathrm{deg}$ (blue solid line) and compared to the corresponding connection length (dashed lines). It can be seen that $n_{e}$ and $T_{e}$ in the magnetic lobes outside the separatrix (at $R=108 \mathrm{~cm}$, index $L$ ) is increased with respect to a toroidal position where no such lobe is present (index $R$ ):

$$
\begin{aligned}
& n_{e, L}=4.2 \cdot 10^{18} \mathrm{~m}^{-3} \\
& n_{e, R}=2.9 \cdot 10^{18} \mathrm{~m}^{-3} \\
& T_{e, L}=68 \mathrm{eV} \\
& T_{e, R}=37 \mathrm{eV}
\end{aligned}
$$

which is an increase by $45 \%$ for $n_{e}$ and $84 \%$ for $T_{e}$. This behavior was also observed in simulations of limiter RMP scenarios at TEXTOR and DIII-D [30]. As the toroidal position of diagnostic equipment in the experiment is fixed, direct comparison to experimental observations remains challenging. However, with a new RMP coil set proposed for DIII-D, it is possible to smoothly rotate the RMP field and hence the magnetic field structure in front of the diagnostics. 
Three-dimensional edge transport simulations for DIII-D plasmas with resonant magnetic perturbations 7
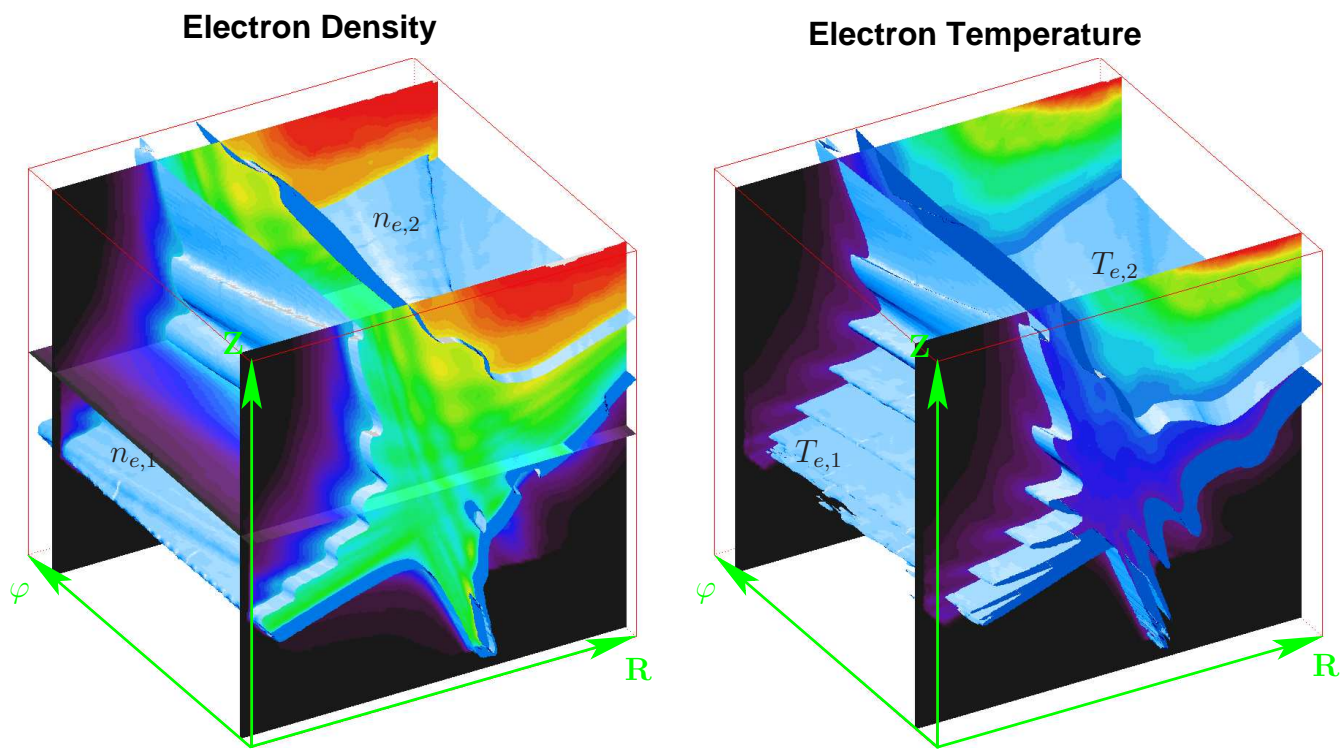

Figure 4. $3 \mathrm{D}$ visualization of electron density (left) and temperature (right) at the X-point region: $R=100 \ldots 160 \mathrm{~cm}, Z=-140 \ldots-82 \mathrm{~cm}, \varphi=$ $-20 \ldots 100$ deg. Iso- $n_{e}$ surfaces are blue-shaded for $n_{e, 1}=4.5 \cdot 10^{18} \mathrm{~m}^{-3}$ and $n_{e, 2}=9 \cdot 10^{18} \mathrm{~m}^{-3} ;$ iso- $T_{e}$ surfaces are at $T_{e, 1}=60 \mathrm{eV}$ and $T_{e, 2}=120 \mathrm{eV}$.
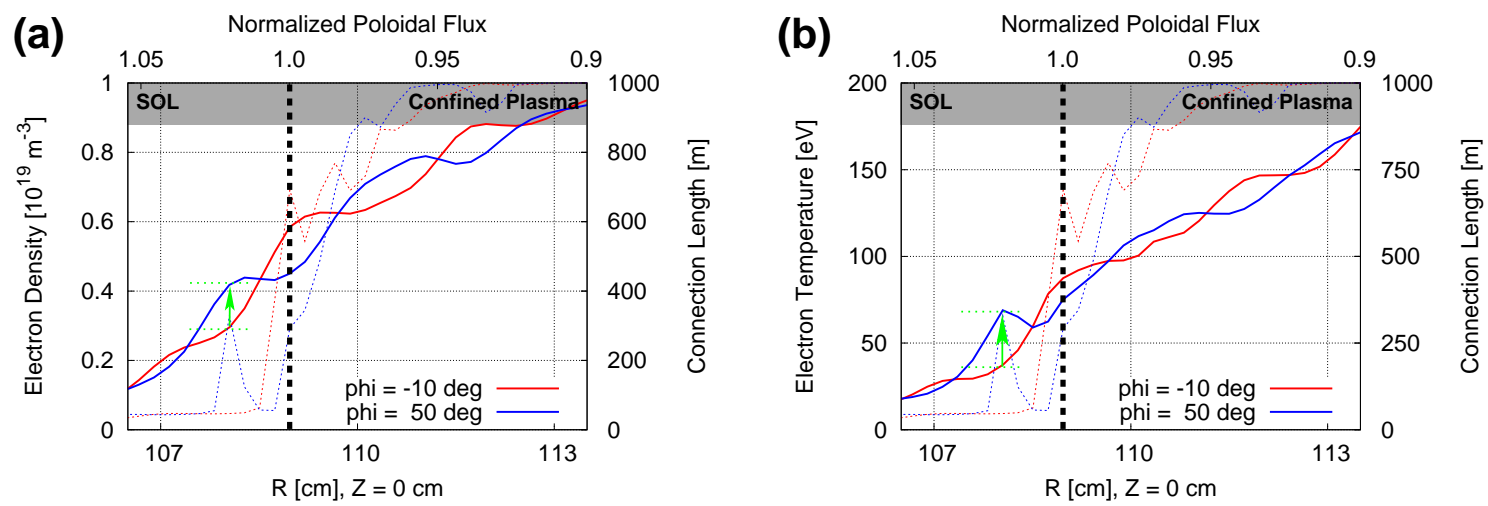

Figure 5. Solid lines: midplane profiles of electron density (a) and temperature (b), dashed lines: connection length. Profiles are taken at $\varphi_{1}=-10 \mathrm{deg}$ (red) and $\varphi_{2}=50 \mathrm{deg}$ (blue). 
Three-dimensional edge transport simulations for DIII-D plasmas with resonant magnetic perturbations 8
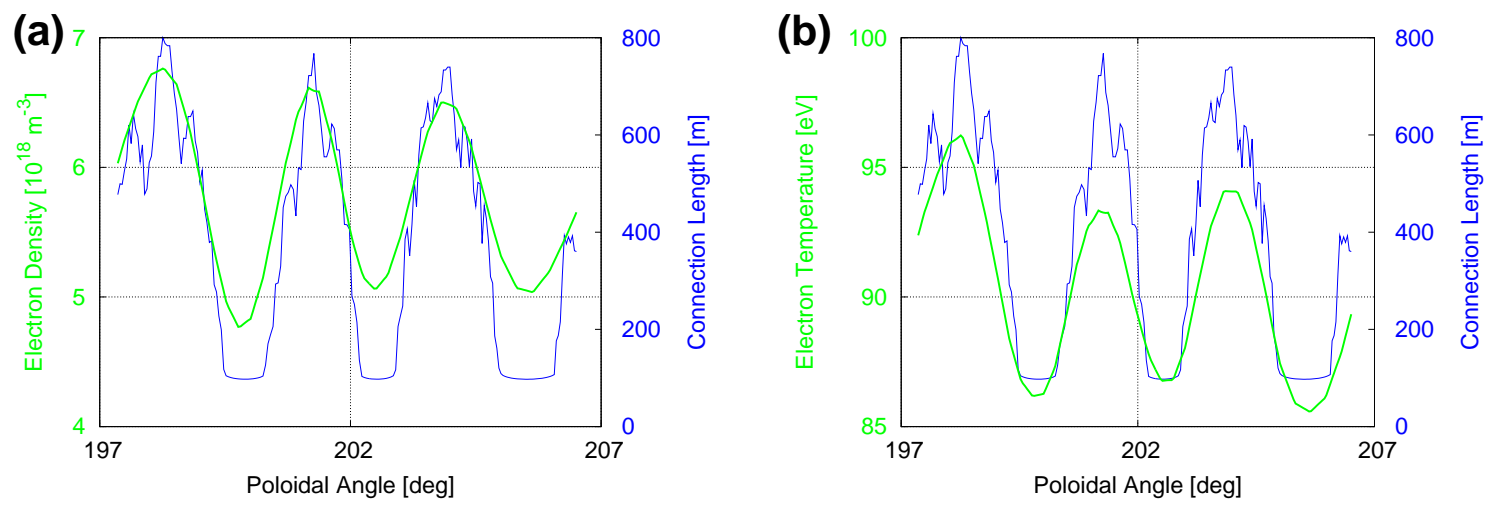

Figure 6. Electron density (a) and temperature (b) on the $\psi^{*}=0.99$ - surface in the $\mathrm{X}$-point region (along the profile marked in figure 1.c).

\subsection{Short magnetic flux tubes}

A mesh of short magnetic flux tubes, i.e. field line bundles with short connection length $L_{c} \approx 100 \mathrm{~m}$, exists inside the separatrix (figure 1.c). These short flux tubes are suspected to act as an additional scrape-off layer (SOL) and hence, as a sink for the plasma [31] which is supported by modeling and experimental observations at the TEXTOR tokamak $[32,11,12]$.

In figure 6 profiles of $n_{e}$ and $T_{e}$ on the $\psi^{*}=0.99$-surface are shown and compared to the connection length of field lines. A strong modulation of $n_{e}$ and $T_{e}$ between short flux tubes (index $F$ ) and long field lines (index $L$ ) is found. Average peak an minimum values are:

$$
\begin{aligned}
& n_{e, F}=5.0 \cdot 10^{18} \mathrm{~m}^{-3} \\
& n_{e, L}=6.5 \cdot 10^{18} \mathrm{~m}^{-3} \\
& T_{e, F}=86 \mathrm{eV} \\
& T_{e, L}=95 \mathrm{eV}
\end{aligned}
$$

which corresponds to a density reduction of $24 \%$ and a temperature reduction of $10 \%$. This is in qualitative agreement with experimental observations and modeling at the TEXTOR tokamak [11, 12], even the more pronounced effect on $n_{e}$. These findings suggests that short flux tubes act like an additional SOL. From figure 1.c it seems that flux tubes have a well-formed cross-section of about $1 \mathrm{~cm} \times 1 \mathrm{~cm}$ and thus, can be described by a 1D SOL model. However, when tracing this cross-section along the flux tube to the divertor plates (figure 7, here only in positive direction), a strong deformation of the shape occurs just after one toroidal turn. The flux tube gets stretched in one direction to more than $30 \mathrm{~cm}$ while in the other direction it is compressed to less than $1 \mathrm{~mm}$, which results from a conservation of toroidal flux in the tube as it connect to different radial positions. After a few toroidal turns the streching relaxes again, but then the flux tube is folded into U-shape after a full poloidal turn. In the end this U-shape is stretched again so that the flux tubes leaves an expanded footprint of $\Delta \vartheta=120 \mathrm{deg}$ in toroidal direction on the inner strike point. 
Three-dimensional edge transport simulations for DIII-D plasmas with resonant magnetic perturbations 9

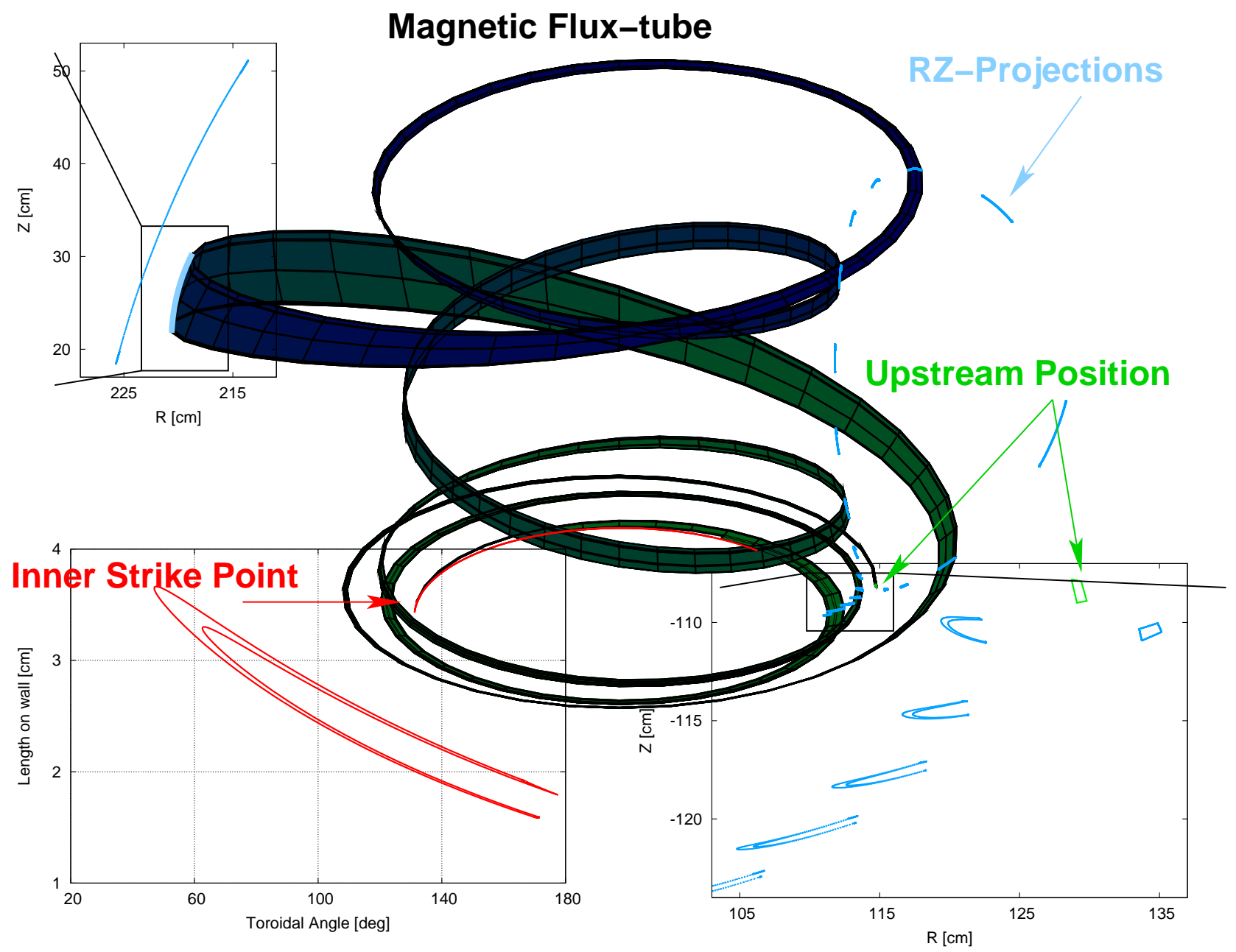

Figure 7. Visualization of a magnetic flux tube. The light blue contours are the updated shape of the flux tube after steps of $\Delta \varphi=120 \mathrm{deg}$. The footprint on the inner strike point is marked in red.

This shape-analysis already suggest that it is not sufficient to put a 1D transport model ontop of these 3D flux tubes. We will demonstrate this now by comparing profiles of $n_{e}, T_{e}$ and mach number $M$ along this flux tube to a $1 \mathrm{D}$ sheath-limited SOL model [33]. Let $x \in[-L, L]$ denote the coordinate along the flux tube with connection length $2 L$, then the isothermal fluid model is:

$$
\begin{aligned}
& n(x)=\frac{n_{0}}{1+M^{2}(x)} \\
& M(x)=\frac{1}{\hat{y}}\left[1-\sqrt{1-\hat{y}^{2}}\right], \quad \hat{y}=\frac{x}{L} \\
& T(x)=T_{0}
\end{aligned}
$$


(a)

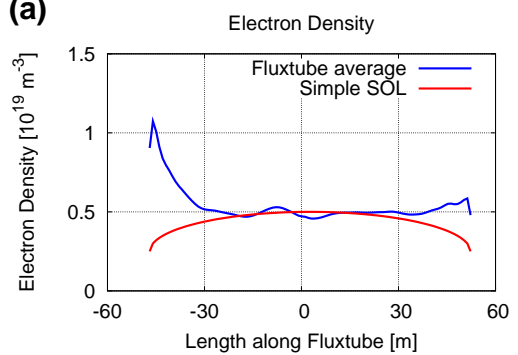

(b)

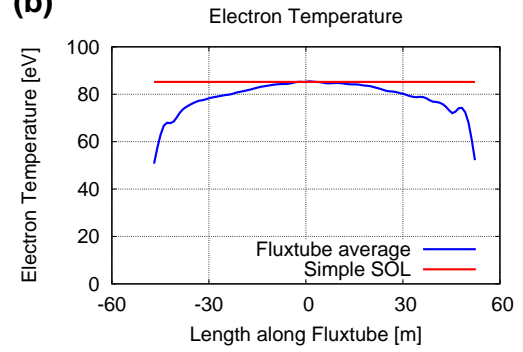

(c)

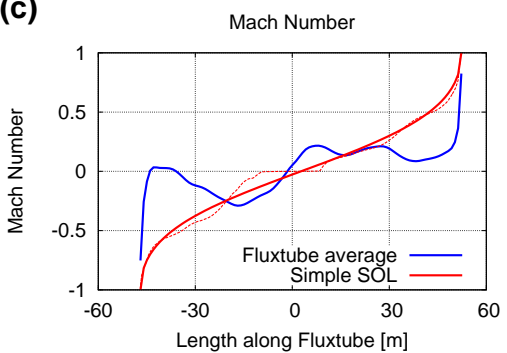

Figure 8. Profiles of (a) $n_{e}$, (b) $T_{e}$ and (c) $M$ along the flux tube from figure 7. 4 field lines $L_{1} \ldots L_{4}$ are selected within the flux tube to calculate a flux tube average (blue line). This is compared to an 1D isothermal fluid model (red line). The red dashed line in (c) is from an extended model with local sources adapted to the flux tube surface.

with free model parameters $n_{0}, T_{0}$ at the upstream position and uniformly distributed sources. Figure 8 shows $n_{e}, T_{e}$ and $M$ profiles along 4 selected field lines $L_{1} \ldots L_{4}$ and an averaged value for the flux tube (blue line). Obviously, the 1D isothermal fluid model (green line) does not reproduce the plasma paremeters in this 3D magnetic flux tube. In particular $T_{e}=$ const along the flux tube is not observed: the upstream value of $T_{e}=85 \mathrm{eV}$ drops to $T_{e}=51$ at the target. A conductionlimited SOL model on the other hand is also not applicable, because we have $M \neq 0$ throughout the flux tube.

The density, on the other hand, increases from an upstream value of $n_{e}=$ $0.5 \cdot 10^{19} \mathrm{~m}^{-3}$ to $n_{e} \approx 1.0 \cdot 10^{19} \mathrm{~m}^{-3}$ at the outer strike point, while it is only slightly increased at the inner strike point. In general, $n_{e}$ and $T_{e}$ profiles bear resemblance to a high-recycling flux tube, however, the strong modulations in $M$ indicates that 3D transport effects are important. In any case, sources are not uniformly distributed along the flux tube. These are due to diffusion from long field lines into the flux tube, but also due to ionization of neutrals. Let us assume for simplicity that the former are proportional to the flux tube surface per toroidal angle $A(\varphi)$, then $n_{e}$ and $M$ in the isothermal fluid model are still determined by (4)-(5), however with $\hat{y}$ replaced by

$$
\hat{y}=\frac{1}{A(\varphi(L))} \int_{0}^{\varphi(x)} d \tilde{\varphi} A(\tilde{\varphi}) .
$$

The modified mach number profile is shown in figure 8 by a red dashed line. This correction slightly changes the $M$ profile, however, it can not explain the results from $3 \mathrm{D}$ modeling. Certainly, the source distribution is more complex when considering 3D transport effects. In particular momentum sources/sinks need to be included in the balance equations. From (4)-(5) with particle sources only it is not possible to obtain a flow reversal and an increase of $n_{e}$ in front of the target. Our 3D edge model includes transport and ionization of neutrals. The resulting effects on the plasma included in 1) by particle, momentum and energy sources $S_{p}, S_{m}$ and $S_{e e}, S_{e i}$, respectively. $S_{p}$ and $S_{m}$ along the flux tube are shown in figure 9. Obviously, neutral particles penetrate into the upstream region of the flux tube and contribute to particle and momentum sources for the plasma. The penetration of neutrals depends on local plasma parameter, and hence, it is an intrinsic 3D transport problem. In conclusion, 
Three-dimensional edge transport simulations for DIII-D plasmas with resonant magnetic perturbations11

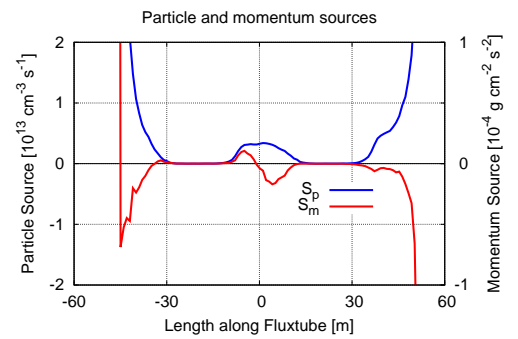

Figure 9. Particle and momentum sources along the flux tube from figure 7 due to plasma interaction with neutral particles.

it is not sufficient to put a 1D transport model ontop of a 3D magnetic geometry, not even for rather simple magnetic flux tubes.

\section{Imprint on the divertor wall}

The magnetic footprint in the presence of RMP fields splits into a characteristic striation pattern (figure 1.d). Particle and heat fluxes to the wall are guided by the underlaying magnetic topology. Hence, a similar striation pattern is expected also for particle and heat flux. These imprints, however, depend on the transport characteristics in the very complex 3D plasma edge and in particular on the level of cross-field transport. The anomalous transport coefficients $D, \chi_{\perp}$ are a priori unkown and hence, are free parameters in our model. Therefore we will give a rough estimate of the correct cross-field transport level in section 5.1 before we turn to a direct comparison to experimental observations in secion 5.2.

\subsection{Estimation of cross-field transport}

In order to estimate the correct level of cross-field transport we perform three independent simulations with $D_{\perp, 1}=1.0 \mathrm{~m}^{2} \mathrm{~s}^{-1}, D_{\perp, 2}=0.25 \mathrm{~m}^{2} \mathrm{~s}^{-1}$ and $D_{\perp, 3}=$ $0.1 \mathrm{~m}^{2} \mathrm{~s}^{-1}$, while in each case $\chi_{\perp, e}=\chi_{\perp, i}=3 D$. These calculations are based on the magnetic configuration of DIII-D discharge 122342 with a simplified wall geometry (i.e. a plain horizontal wall). Simulation results of particle and heat flux onto the divertor target $\Gamma_{\text {target }}$ and $q_{\text {target }}$, respectively, are presented in figure 10. Both $\Gamma_{\text {target }}$ and $q_{\text {target }}$ show a clear helical pattern for all transport levels. However, a sharp striation pattern is present only for low enough cross-field transport $\left(D_{\perp} \lesssim 0.25 \mathrm{~m}^{2} \mathrm{~s}^{-1}\right)$. Such a distinct striation pattern is observerd in the experiment $[24,13]$ for the particle flux. Hence, $D_{\perp, 2}$ provides an upper limit for the free model parameter for cross-field diffusion as such a blurred pattern of over up to $15 \mathrm{~cm}$ along the wall, as emerges with $D_{\perp, 1}$, is not observed experimentally. Remarkably, only very weak striation patterns are observed for the heat flux in low density RMP experiments such as DIII-D discharges 122342 and 132731 [34] while heat flux splitting is observed in high density DIII-D RMP experiments [35]. The peak heat flux is located just at the strike point, consistent with low values of $\chi_{\perp, e}, \chi_{\perp, i}$. The presence of a clear striation pattern in the corresponding simulation results, however, indicates some missing physical processes in the present transport model when considering the difference between high and low density plasmas. However, the same qualitative behavior was also found in earlier 


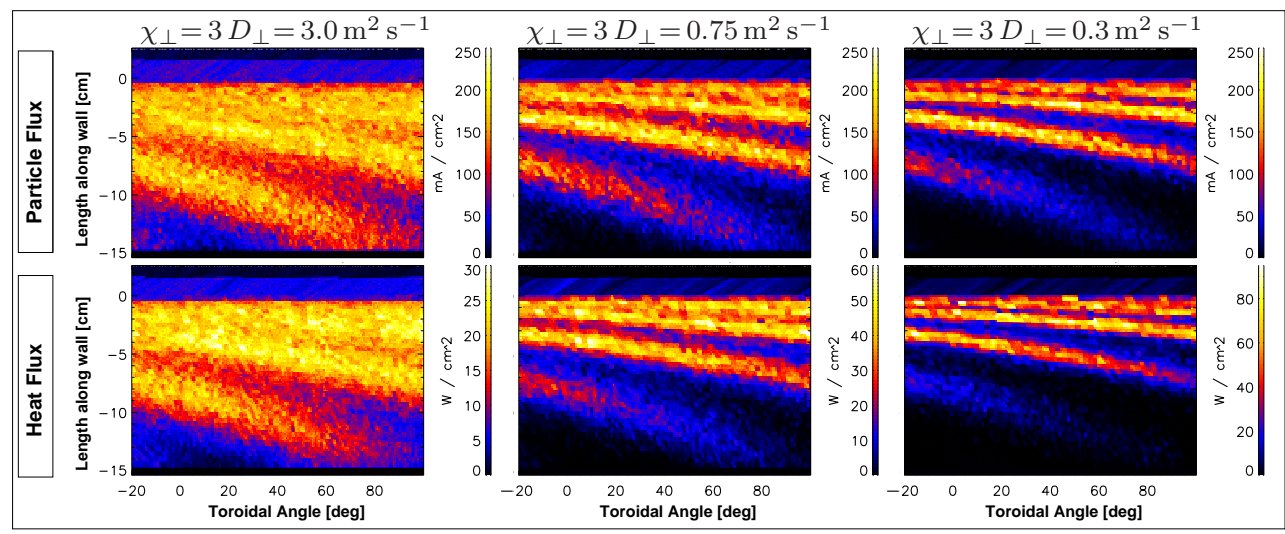

Figure 10. Particle (upper row) and heat flux (lower row) footprints at the outer strike point for three levels of cross-field transport. The magnetic configuration is based on DIII-D discharge 122342 under the assumption of a plain horizontal wall (e.g. neglecting the 45 deg tile at the high field side).

heat transport simulations with the E3D code [36]. Our simulations now demonstrate that this mismatch cannot be explained by the inclusion of self-consistent particle and momentum transport as well as recycling neutrals. To validate the code for the purpose target particle and heat load modeling we now perform a more detailed analysis.

\subsection{Analysis of target particle and heat fluxes}

For a direct comparison to experimental observations we investigate the more recent DIII-D discharge 132731, where we included the complete wall geometry in our simulations. Here we use transport coefficients of $D_{\perp}=0.2 \mathrm{~m}^{2} \mathrm{~s}^{-1}$ and $\chi_{\perp, e}=\chi_{\perp, i}=$ $0.6 \mathrm{~m}^{2} \mathrm{~s}^{-1}$, slightly below the uppler limit estimated above. The input power is here set to $P_{\text {in }}=6.3 \mathrm{MW}$ which corresponds to the experimental value during the $\mathrm{H}$-mode phase. Profiles of the arising target particle and heat fluxes at the inner strike point are shown in figure 11. Both profiles are well alligned to the magnetic topology (black dashed lines). The modeled particle flux (blue) is compared to the observed $D_{\alpha}$ emission (green) which is correlated to the experimental particle flux. Both profiles show a clear splitting in qualitative agreement. The slight shifts in the two outer peaks are probably due to error fields (e.g. misalignments of the field coils) which were neglected in our simulations. The peak particle flux of $\Gamma_{1}=195 \mathrm{~mA} \mathrm{~cm}^{-2}$ is reduced to $\Gamma_{2}=150 \mathrm{~mA} \mathrm{~cm}^{-2}$ at the first and $\Gamma_{3}=90 \mathrm{~mA} \mathrm{~cm}^{-2}$ at the second minimum. The outer peak is with $\Gamma_{4}=155 \mathrm{~mA} \mathrm{~cm}^{-2}$ at $80 \%$ of $\Gamma_{1}$. A similar splitting is found for the modeled heat flux (figure 11.b) which remains after including the complete wall geometry. The experimental peak heat flux of $q_{1, \exp }=3.2 \mathrm{MW} \mathrm{m}^{-2}$ drops to $q_{3, \exp }=0.5 \mathrm{MW} \mathrm{m}^{-2}$ without any significant splitting. The modeled heat flux is with $q_{1}=4.7 \mathrm{MW} \mathrm{m}^{-2}$ and $q_{2}=5.3 \mathrm{MW} \mathrm{m}^{-2}$ much larger than the observed one. Even the ratio of the second miminum $q_{3}=1.6 \mathrm{MW} \mathrm{m}^{-2}$ to $q_{1}$ is with $35 \%$ significantly larger then the observed one of $q_{3, \exp } / q_{1, \exp }=16 \%$. The presence of a such a striation pattern in the modeled heaet flux indicates some missing physical processes in the present transport model. In particular kinetic corrections to the fluid model, which have recently been investigated at the TEXTOR tokamak [20], might be necessary. In 

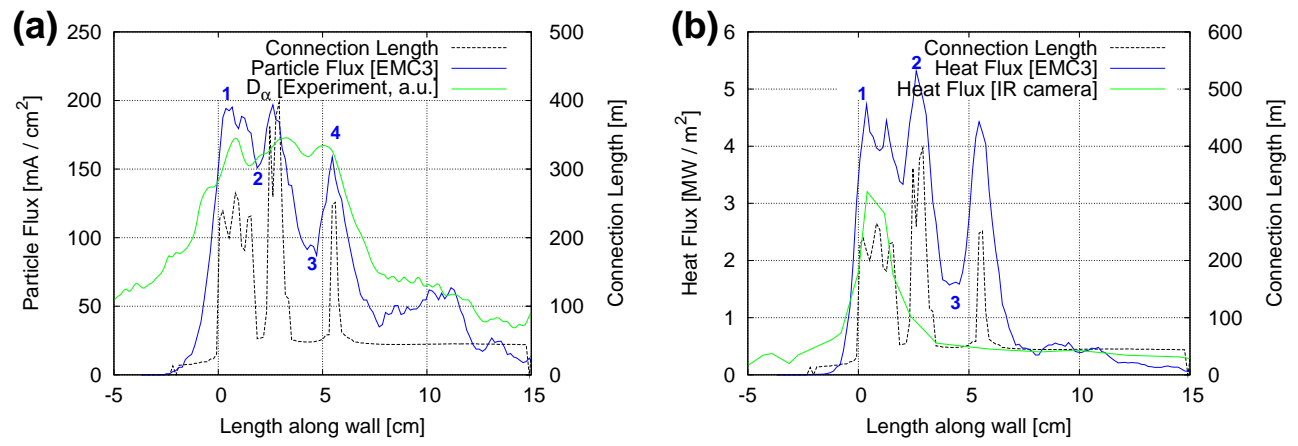

Figure 11. Profiles of target particle (a) and heat fluxes (b) in comparison to experimental observations.

addition, the treatment of cross-field transport in our model needs to be advanced to include effects of an edge transport barrier in $\mathrm{H}$-mode plasmas. Such corrections have been taken into account in other approaches [10], there however without the full 3D magnetic topology, and are here left for future analysis.

\section{Conclusions}

Self-consistent calculations of particle, parallel momentum and energy transport in the edge plasma were performed, including recycling neutrals. We demonstrated that a certain low level of anomalous cross-field transport must not be exceeded in order to obtain pronounced striation pattern in target particle and heat fluxes. The modeled particle flux is found to be in qualitative agreement to experimental observations, the modeld heat flux, however, also shows a clear striation pattern in mismatch to the corresponding observations. This mismatch indicates the necessity to include kinetic corrections of the electron heat flux as well as an advancement of the cross-field transport model to include effects of an edge transport barrier in H-mode plasmas. In section 4 we demonstrated the strong $3 \mathrm{D}$ effects of RMPs on the plasma, in particular that the structure of helical magnetic lobes is very well reflected in the plasma parameters $n_{e}$ and $T_{e}$. By means of a short magnetic flux tube we demonstrated that it is not sufficient to put a $1 \mathrm{D}$ transport model ontop of a 3D magnetic geometry but it is necessary to include the full 3D transport. The poloidal modulation found in the region of these flux tube is in qualitative agreement to observations and modeling at the TEXTOR tokamak.

\section{Acknowledgements}

The presented computer simulations were performed on an IBM Power 6 cluster at Forschungszentrum Jülich and Rechenzentrum Garching, the latter within the Euforia project.

\section{References}

[1] G.T.A. Huysmans. ELMs: MHD instabilities at the transport barrier. Plasma Phys. Control. Fusion, 47:B165-B178, 2005. 
Three-dimensional edge transport simulations for DIII-D plasmas with resonant magnetic perturbations14

[2] A Loarte, M Becoulet, G Saibene, R Sartori, D J Campbell, T Eich, A Herrmann, M Laux, W Suttrop, B Alper, P J Lomas, G Matthews, S Jachmich adn J Ongena, P Innocente, and EFDA-JET Workprogramme Collaborators. Characteristics and scaling of energy and particle losses during Type I ELMs in JET H-modes. Plasma Phys. Control. Fusion, 44:1815, 2002.

[3] T.E. Evans, R.A. Moyer, P.R. Thomas, J.G. Watkins, T.H. Osborne, J.A. Boedo, E.J. Doyle, M.E. Fenstermacher, K.H. Finken, R.J. Groebner, M.Groth, J.H. Harris, R.J. La Haye, C.J. Lasnier, S. Masuzaki, N. Ohyabu, D.G. Pretty, T.L. Rhodes, H. Reimerdes, D.L. Rudakov, M.J. Schaffer, G. Wang, and L. Zeng. Suppression of Large Edge-Localized Modes in High-Confinement DIII-D Plasmas with a Stochastic Magnetic Boundary. Phys. Rev. Lett., 92(23):(235003), 2004.

[4] T.E. Evans, R.A. Moyer, K.H. Burrell, M.E. Fenstermacher, I. Joseph, A.W. Leonard, T.H. Osborne, G.D. Porter, M.J. Schaffer, P.B. Snyder, P.R. Thomas, J.G. Watkins, and W.P. West. Edge stability and transport control with resonant magnetic perturbations in collisionless tokamak plasmas. Nature Physics, 2:419-423, 2006.

[5] Y. Liang, H. R. Koslowski, P. R. Thomas, E. Nardon, B. Alper, P. Andrew, Y. Andrew, Arnoux, Y. Baranov, M. Becoulet, M. Beurskens, T. Biewer, M. Bigi, K. Crombe, E. De La Luna, P. de Vries, W. Fundamenski, S. Gerasimov, C. Giroud, M. P. Gryaznevich, N. Hawkes, S. Hotchin, D. Howell, S. Jachmich, V. Kiptily, L. Moreira, V. Parail, S. D. Pinches, E. Rachlew, and O. Zimmermann. Active Control of Type-I Edge-Localized Modes with n=1 Perturbation Fields in the JET Tokamak. Phys. Rev. Lett., 98(265004):1-5, 2007.

[6] Ph. Ghendrih, A. Grosman, and H. Capes. Theoretical and experimental investigations of stochastic boundaries in tokamaks. Plasma Phys. Control. Fusion, 38:1653, 1996.

[7] Ph. Ghendrih, M. Becoulet, L. Colas, A. Grosman, R. Guirlet, J. Gunn, T. Loarer, A. Azeroual, V. Basiuk, B. Beaumont, A. Becoulet, P. Beyer, S. Bremond, J. Bucalossi, H. Capes, Y. Corre, L. Costanzo, C. De Michelis, P. Devynck, S. Feron, C. Friant, X. Garbet, R. Giannella, C. Grisolia, W. Hess, J. Hogan, L. Ladurelle, F. Laugier, G. Martin, M. Mattioli, B. Meslin, P. Monier-Garbet, D. Moulin, F. Nguyen, J.-Y. Pascal, A.-L. Pecquet, B. Pegourie, R. Reichle, F. Saint-Laurent, J.-C. Vallet, M. Zabiego, and Tore Supra Team. Progress in ergodic divertor operation on Tore Supra. Nuclear Fusion, 42(10):1221-1250, 2002.

[8] P.B. Snyder, H.R. Wilson, J.R. Ferron, L.L. Lao, A.W. Leonard, T.H. Osborne, A.D. Turnbull, D. Mossessian, M. Murakami, and X.Q. Xu. Edge localized modes and the pedestal: A model based on coupled peeling-ballooning modes. Phys. Plasmas, 9:2037, 2002.

[9] M. Z. Tokar, T. E. Evans, A. Gupta, R. Singh, P. Kaw, , and R. C. Wolf. Mechanisms of Edge-Localized-Mode Mitigation by External-Magnetic-Field Perturbations. Phys. Rev. Lett., 98:095001 (1-4), 2007.

[10] M.Z. Tokar, T.E. Evans, A. Gupta, D. Kalupin, A. Nicolai, R. Singh, and B. Unterberg. Modelling of pedestal transport during ELM suppression by external magnetic field perturbations. Nuclear Fusion, 48:024006, 2008.

[11] M.W. Jakubowski, O. Schmitz, S.S. Abdullaev, S. Brezinsek, K.H. Finken, A. Krämer-Flecken, M. Lehnen, U. Samm, K.H. Spatschek, B. Unterberg, R.C. Wolf, and TEXTOR Team. Change of the Magnetic-Field Topology by an Ergodic Divertor and the Effect on the Plasma Structure and Transport. Phys. Rev. Lett., 96:(035004), 2006.

[12] O. Schmitz, M.W. Jakubowski, H. Frerichs, D. Harting, M. Lehnen, B. Unterberg, S.S. Abduallaev, S. Brezinsek, I. Classen, T. Evans, Y. Feng, K.H. Finken, M. Kantor, D. Reiter, U. Samm, B. Schweer, G. Sergienko, G.W. Spakman, M. Tokar, E. Uzgel, R.C. Wolf, and the TEXTOR Team. Identification and analysis of transport domains in the stochastic boundary of TEXTOR-DED for different mode spectra. Nuclear Fusion, 48:024009, 2008.

[13] O. Schmitz, T.E. Evans, M.E. Fenstermacher, H. Frerichs, M.W. Jakubowski, M.J. Schaffer, A. Wingen, W.P. West, N.H. Brooks, K.H. Burrell, J.S. deGrassie, Y. Feng, K.H. Finken, P. Gohil, M. Groth, I. Joseph, C.J. Lasnier, M. Lehnen, A.W. Leonard, S. Mordijck, R.A. Moyer, A. Nicolai, T.H. Osborne, D. Reiter, U. Samm, K.H. Spatschek, H. Stoschus, B. Unterberg, E.A. Unterberg, J.G. Watkins, R. Wolf, and the DIII-D and TEXTOR Teams. Three dimensional transport analysis for ELM control experiments in ITER similar shape plasmas at low collisionality in DIII-D. Plasma Phys. Control. Fusion, 50:124029, 2008.

[14] Y. Feng, F. Sardei, J. Kisslinger, and P. Grigull. A 3D Monte Carlo code for plasma transport in island divertors. Journal of Nuclear Materials, 241-243:930-934, 1997.

[15] Y. Feng, F. Sardei, and J. Kisslinger. 3D fluid modelling of the edge plasma by means of a Monte Carlo technique. Journal of Nuclear Materials, 266-269:812-818, 1999.

[16] Y. Feng, J. Kisslinger, and F. Sardei. Formulation of a Monte Carlo model for edge plasma transport. In ECA, editor, 27th EPS Conference on Contr. Fusion and Plasma Phys., volume 
Three-dimensional edge transport simulations for DIII-D plasmas with resonant magnetic perturbations15

24B, pages 1188-1191, Budapest, 12-16 June 2000.

[17] D. Reiter, M. Baelmans, and P. Boerner. The EIRENE and B2-EIRENE codes. Fusion Science and Technology, 47:172, 2005.

[18] M. Kobayashi, Y. Feng, F. Sardei, D. Reiter, D. Reiser, and K.H. Finken. Implementation of the EMC3-EIRENE code on TEXTOR-DED: accuracy and convergence study. Contrib. Plasma Phys., 44(1-3):25-30, 2004.

[19] M. Kobayashi, Y. Feng, F. Sardei, D. Reiter, K.H. Finken, and D. Reiser. 3D numerical transport study of the edge ergodized plasma in TEXTOR-DED. Nuclear Fusion, 44:S64-S73, 2004.

[20] D. Harting, D. Reiter, Y. Feng, O. Schmitz, and H. Frerichs. 3D edge transport studies with EMC3-EIRENE for the Dynamic Ergodic Divertor (DED) at TEXTOR. Contrib. Plasma Phys., 48(1-3):1-7, 2008.

[21] M. Kobayashi, Y. Feng, A. Loarte, G. Federici, G. Strohmayer, M. Shimada, F. Sardei, D. Reiter, and M. Sugihara. 3D edge transport analysis of ITER start-up configuration for limiter power load assessment. Nuclear Fusion, 47:61-73, 2007.

[22] H. Frerichs, D. Reiter, Y. Feng, and D. Harting. Block-structured grids in Lagrangian 3D edge plasma transport simulations. Comp. Phys. Commun., (submitted), 2009.

[23] L.L. Lao, H.St. John, R.D. Stambaugh, A.G. Kellman, and W. Pfeiffer. Reconstruction of current profile parameters and plasma shapes in tokamaks. Nuclear Fusion, 25:1611-1622, 1985.

[24] T.E. Evans. Chaos, Complexity and Transport: Theory and Applications. World Scientific Press, 2008.

[25] A. Wingen, T.E. Evans, and K.H. Spatschek. Footprint structures due to resonant magnetic perturbations in DIII-D. Phys. Plasmas, 16:042504, 2009.

[26] T.E. Evans, R.K.W. Roeder, J.A. Carter, B.I. Rapoport, M.E. Fenstermacher, and C.J. Lasnier. Experimental signatures of homoclinic tangles in poloidally diverted tokamaks. J. Phys. Conf. Ser., 7:174, 2005.

[27] J. Guckenheimer and P. Holmes. Nonlinear Oscillations, Dynamical Systems, and Bifurcations of Vector Fields. Applied Mathematical Science, 1983.

[28] Y. Feng, F. Sardei, and J. Kisslinger. A simple highly accurate field-line mapping technique for three-dimensional Monte Carlo modeling of plasma edge transport. Phys. Plasmas, 12(052505):1-7, 2005.

[29] S.I. Braginskii. Transport processes in a plasma. Review of Plasma Physics, 1:205-311, 1965.

[30] H.G. Frerichs, O. Schmitz, D. Harting, D. Reiter, B. Unterberg, Y. Feng, T.E. Evans, M.E. Fenstermacher, I. Joseph, and R.A. Moyer. 3D numerical analysis of magnetic topology and edge transport for RMP limiter scenarios at TEXTOR and DIII-D. In Proc. 35th EPS Conf. Plasma Phys., number P2.009, 2008.

[31] F. Nguyen, P. Ghendrih, and A. Grosman. Interaction of stochastic boundary layer with plasma facing components. Nuclear Fusion, 37(6):743-757, 1997.

[32] T. Eich, D. Reiser, and K.H. Finken. Two dimensional modelling approach to transport properties of the TEXTOR-DED laminar zone. Nuclear Fusion, 40(10):1757-1772, 2000.

[33] P.C. Stangeby. The Plasma Boundary of Magnetic Fusion Devices. Institute of Physics Publishing Bristol and Philadelphia, 2000.

[34] M.W. Jakubowski, T.E. Evans, M.E. Fenstermacher, M. Groth, C.J. Lasnier, A.W. Leonard, O. Schmitz, J.G. Watkins, T. Eich, W. Fundamenski, R.A. Moyer, R.C. Wolf, L.B. Baylor, J.A. Boedo, K.H. Burrell, H. Frerichs, J.S. deGrassie, P. Gohil, I. Joseph, S. Mordijck, M. Lehnen, C.C. Petty, R.I. Pinsker, D. Reiter, T.L. Rhodes, U. Samm, M.J. Schaffer, P.B. Snyder, H. Stoschus, T. Osborne, B. Unterberg, E. Unterberg, and W.P. West. Overview of the results on divertor heat loads in RMP controlled H-mode plasmas on DIII-D. Nuclear Fusion, (submitted), 2009.

[35] T.E. Evans, I. Joseph, R.A. Moyer, M.E. Fenstermacher, C.J. Lasnier, and L.W. Yan. Experimental and numerical studies of separatrix splitting and magnetic footprints in DIII-D. J. Nucl. Mater., 363-365:570, 2007.

[36] I. Joseph, T.E. Evans, A.M. Runov, M.E. Fenstermacher, M. Groth, S.V. Kasilov, C.J. Lasnier, R.A. Moyer, G.D. Porter, M.J. Schaffer, R. Schneider, and J.G. Watkins. Calculation of stochastic thermal transport due to resonant magnetic perturbations in diii-d. Nuclear Fusion, 48:045009, 2008. 